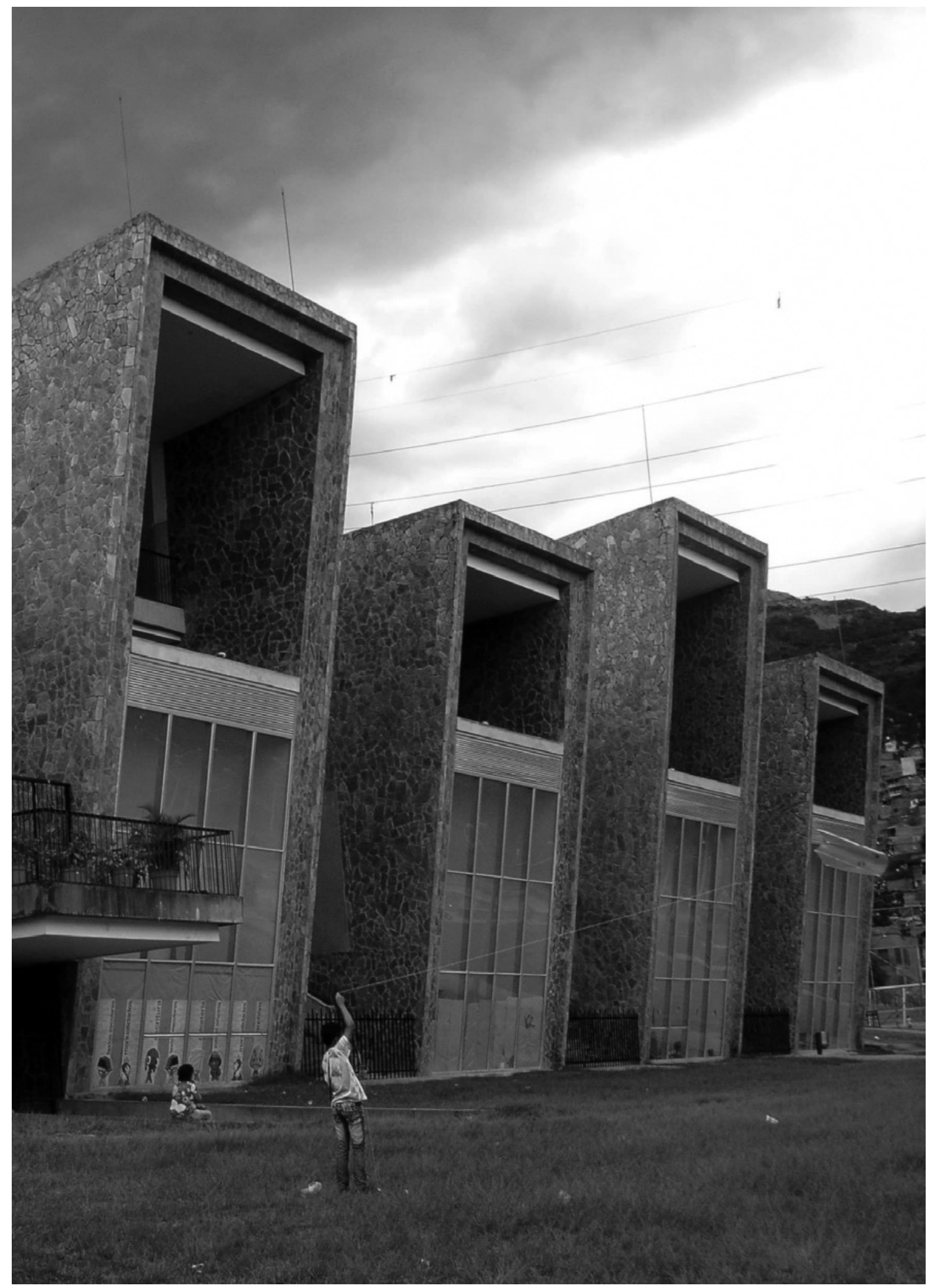

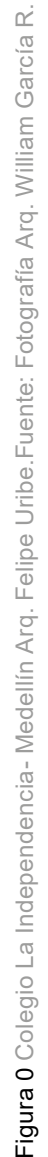



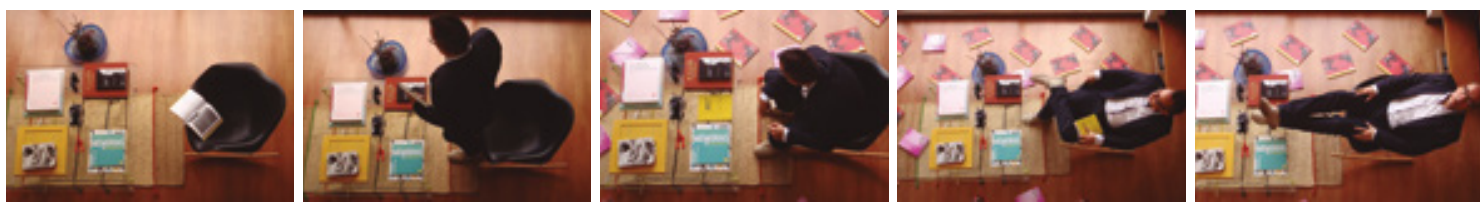

Secuencia: En casa del arquitecto William García R.

Fotos: Jorge Enrique García Quintero

\title{
PEDAGOGÍAS DE UNA IDEOLOGÍA: ARQUITECTURA EDUCATIVA EN COLOMBIA (1994-2016)'
}

\author{
PEDAGOGIES OF AN IDEOLOGY: EDUCATIONAL ARCHITECTURE IN COLOMBIA (1994- \\ 2016)
}

\section{William García Ramírez²}

\section{RESUMEN}

Este artículo presenta un panorama conceptual de la arquitectura educativa pública en Colombia (1994-2016), poniendo énfasis en la ideología y la pedagogía establecidas por el gobierno colombiano. A través de este panorama se busca visibilizar las principales estrategias proyectuales con las que los arquitectos han materializado una concepción de la educación pública y una visión ideológica del ciudadano colombiano del siglo XXI. La metodología establecida permitió una lectura cruzada entre fuentes documentales (leyes, decretos, y modelos pedagógicos) y planimetrías de los proyectos, una lectura que se enriqueció con la visita presencial a los colegios particularmente estudiados. Por otra parte, el periodo seleccionado (1994-2016) hizo posible elaborar unas conclusiones de orden comparativo, a fin de evidenciar un proceso y una evolución de este tipo de arquitectura en Colombia.

Palabras clave: arquitectura educacional, teoría, ideología, Colombia, pedagogía.

\section{ABSTRACT}

This article presents a conceptual panorama of the public educational architecture in Colombia (1994-2016), emphasizing the ideology and pedagogy established by the Colombian government. Through this panorama, the main project strategies with which the architects have materialized a conceptualization of public education, and an ideological vision of the Colombian citizen of the 21st century are shown. The methodology involved a cross-referenced reading of documentary sources (laws, decrees, and pedagogical models) and project plans, which was enriched by an on-site visit to the specific schools studied. Alternately, the selected period (1994-2016) made it possible to draw comparative conclusions, in order to demonstrate the process and evolution of this type of architecture in Colombia.

Keywords: educational architecture, theory, ideology, Colombia, pedagogy.

Artículo recibido el 30 de agosto de 2017 y aceptado el 21 de diciembre de 2017 DOI: https://doi.org/10.22320/07196466.2017.35.052.07

[1] Este artículo está basado en los resultados de la investigación "Análisis de las memorias descriptivas y planimetrías de proyecto arquitectónico en las Bienales Colombianas de Arquitectura Fase I: Arquitectura Educativa (1990-2010)". Investigador principal: William García R. 2017, Código proyecto: IDPRY7097. Institución patrocinadora: Pontificia Universidad Javeriana.

[2] Facultad de Arquitectura y Diseño, Pontificia Universidad Javeriana, Bogotá, Colombia. Correo: william.garcia@javeriana.edu.co 


\section{INTRODUCCIÓN}

En las últimas dos décadas, la arquitectura educativa en Colombia ha dejado de ser un campo en vías de desarrollo para consolidarse en una realidad incuestionable: El diseño y realización de nuevos equipamientos escolares de carácter público, así como la construcción y ampliación de campus universitarios privados, dominan el panorama de la arquitectura en Colombia de comienzos del siglo XXI. Dentro de este amplio espectro de obras, se destacan los esfuerzos del estado colombiano por proveer de espacios educativos mediante la realización de concursos de arquitectura, con los que se ha venido respondiendo a problemáticas específicas de las diferentes regiones que integran el territorio colombiano, esfuerzos que por su alta calidad han logrado reconocimiento en el medio colombiano. No en vano los ganadores de las 4 últimas Bienales Colombianas de Arquitectura (2010, 2012, 2014 y 2016) corresponden al diseño de arquitectura educativa pública construida en Medellín, Cartagena, Vigía del Fuerte y Bogotá, respectivamente; la concepción de estos proyectos, ubicados en lugares tan disímiles, revelan en sus planteamientos ideas y conceptos teóricos tan significativos como contrastantes entre sí. Esta diversidad llevó a plantear una investigación que profundizara específicamente en la arquitectura educativa pública en Colombia bajo una hipótesis central: Desde sus inicios, el diseño arquitectónico de los colegios y escuelas públicas en Colombia, se puede explicar a partir de una triada conceptual integrada por la relación entre: la ideología de un estado gobernante, el modelo pedagógico adoptado por este gobierno y la estrategia proyectual asumida por el arquitecto frente a este modelo pedagógico y a esta ideología. A partir del desarrollo de esta hipótesis, este artículo presenta un modelo interpretativo de la evolución de la arquitectura educativa en Colombia, sustentado en el análisis de las políticas educativas en relación con los modelos pedagógicos adoptados por el Ministerio de Educación de Colombia, con el fin evidenciar un panorama de las distintas aproximaciones en los modos de pensar y proyectar este tipo de arquitectura a finales del siglo XX y comienzos del siglo XXI. 
En general, las investigaciones historiográficas y teóricas sobre la arquitectura educativa en Colombia coinciden en relacionar los aspectos pedagógicos y arquitectónicos como base argumentativa. Es así como el libro Historia de la arquitectura escolar en Colombia (Maldonado, 1999) perfila una visión historiográfica de la arquitectura educativa, entrelazando las distintas pedagogías adoptadas a lo largo del tiempo con tipologías escolares, utilizadas en colegios públicos y privados. En el campo de las investigaciones teóricas, el enfoque Pedagogía Arquitectura se mantiene, solo que ahora desde la dimensión conceptual. Se trata de investigaciones acerca de los distintos modos de concebir lo arquitectónico como respuesta del arquitecto ante una forma de aprendizaje. Ejemplo de lo anterior son dos de las últimas investigaciones teóricas realizadas en el país: la primera de ellas, adelantada por los investigadores del Observatorio de arquitectura y urbanismo contemporáneos de la Universidad del Valle y titulada Arquitectura y Pedagogía: El Edificio Escolar, se aboca, en gran parte, al estudio historiográfico de arquitecturas educativas propias del movimiento moderno, y se centra en el enfoque Pedagogía - Arquitectura, tal como uno de sus autores afirma: "Este trabajo, (...), recoge las propuestas más significativas en la relación entre programas pedagógicos y formas y espacios arquitectónicos ..." (Ramírez, 2009:31)". Empero, un grupo minoritario de los investigadores se orientó hacia el estudio de los aportes sociales de la arquitectura educativa a la ciudad y al análisis de la normativa legal y los criterios de diseño en la arquitectura educativa; temas contextualizados en casos de arquitecturas más recientes.

La segunda investigación de corte teórico, denominada Construyendo pedagogía. Estándares básicos para construcciones escolares (Rivera Realpe \& Asociados, 2000), evidencia desde el propio título, su compromiso con la relación pedagogía - arquitectura, como estrategia para proponer una serie de criterios arquitectónicos y urbanísticos para el diseño y construcción de equipamientos escolares públicos en Bogotá. Sin embargo, una lectura crítica de esta propuesta, permitió establecer una fuerte influencia de las políticas y promesas de campaña del entonces alcalde de Bogotá, Enrique Peñalosa (1998-2002), en esta investigación y a la postre, en las directrices que posteriormente tomaría la Secretaria de Educación de Bogotá, influencia fundamentalmente expresada en una serie de normas y decretos de orden educativo, los cuales a su vez obedecían a la Ley general de educación de 1994. Estas influencias hicieron evidente la importancia de ampliar el marco conceptual de la investigación más allá de la recurrente relación Pedagogía - Arquitectura, para incluir la dimensión Ideológica como nueva variable conceptual y punto de partida explicativo tanto de los modelos pedagógicos adoptados, como de las arquitecturas construidas. De esta manera, la triada Ideología - Pedagogía - Arquitectura, permite una lectura transversal de las ideas y conceptos que desde distintas disciplinas integran una explicación del panorama de la arquitectura educativa en Colombia de comienzos del siglo XXI.

\section{MÉTODO}

Inicialmente, se partió de un análisis de las memorias descriptivas de los proyectos, así como de las normativas y decretos de orden nacional bajo los cuales se ha venido orientando los modelos pedagógicos en la educación pública, textos que estructuraron una primera base documental, entendida como estrategias conceptuales. Luego, se buscó verificar la aplicación efectiva de estos preceptos en las obras y proyectos construidos en el país, con el fin de establecer las influencias político-pedagógicas en la arquitectura educativa. Para este efecto, la selección de proyectos se realizó a partir de dichas estrategias conceptuales, fruto de una lectura cruzada entre la ideología política establecida en los últimos gobiernos y los modelos pedagógicos implementados en concordancia, cuyo fin fue determinar estudios de caso arquitectónicos que expresaran espacialmente estos conceptos y a través de los cuales se pudiese verificar la hipótesis principal de la investigación. Del universo total de casos revisados en la investigación (96 casos), los primeros escogidos se filtraron a través de las Bienales Colombianas de Arquitectura (Sociedad Colombiana de Arquitectos, 1992 - 2016) y los concursos públicos que sobre arquitectura educativa se han promulgado en el país, como mecanismos de juzgamiento independientes de la arquitectura. A partir de este proceso, se seleccionó, finalmente, proyectos arquitectónicos de cierta significancia y calidad en el país, lo que dio como resultado una concentración de proyectos en los departamentos de Antioquia y la ciudad de Bogotá.

De esta manera, la investigación se dedicó a rastrear en el umbral de los siglos XX y XXI las tres dimensiones mencionadas (ideología, pedagogía, y arquitecturas educativas), con el propósito de cotejarlas en momentos claves de la historia reciente de la arquitectura educativa y en cuya lectura transversal se pudiera perfilar un panorama de la evolución teórico conceptual de este tipo de arquitectura.

\section{Dimensión ideológica: Neoliberalismo}

A finales del siglo pasado, la ideología neoliberal que orienta al gobierno nacional, se centra en el reconocimiento de la diversidad de pensamiento, cultura e identidad de las personas que integran la sociedad colombiana. Esta política educativa es producto de una reflexión ideológica: la Constitución política de Colombia del año 1991, una constitución "Fundada en el respeto de la dignidad humana, en el trabajo y la solidaridad de las personas que la integran y en la prevalencia del interés general" (Ley General de Educación, 1994: Art 1); de allí que sea considerada por muchos como una constitución personalista. Esta marcada orientación ideológica hacia las personas, se verá reivindicada en la definición misma de educación, propuesta en la nueva Ley General de Educación de 1994, y entendida como: "un proceso de formación permanente, personal, cultural y social que se fundamenta en una concepción integral de la persona humana, de su dignidad, de sus derechos $y$ de sus deberes" (ídem). 
Esta influencia del personalismo deriva en una ampliación en el alcance de la educación en Colombia, más allá de una cultura académica, para alcanzar una cultura de lo ciudadano, pues bajo esta nueva ley se promueve: "La formación en el respeto a la vida y demás derechos humanos, a la paz, a los principios democráticos de convivencia, pluralismo, justicia, solidaridad y equidad, y también en el ejercicio de la tolerancia y la libertad" (Ibídem, Art 5). De esta manera, la educación en Colombia se propone como mecanismo capaz de articular en el estudiante, una formación de competencias académicas y el desarrollo de habilidades sociales en pro de una sana convivencia.

\section{Dimensión pedagógica: Pedagogía personalista}

En términos pedagógicos, el personalismo es una estrategia de formación educativa adaptable a las realidades culturales, sociales y económicas propias y diferentes de cada región, de cada ciudad, y de cada barrio en Colombia. Esta estrategia expresada en la Ley General de Educación a través del Proyecto Educativo Institucional o P.E.I., tiene hasta el día de hoy la responsabilidad de expresar "La forma como se ha decidido alcanzar los fines de la educación definidos por la ley, teniendo en cuenta las condiciones sociales, económicas y culturales de su medio" (Decreto 3860, 1994: Art 14). Por lo que su interpretación a través de diversas estrategias arquitectónicas, ha buscado responder a necesidades particulares de cada región, estrategias entre las que se destacan aquellas que se exponen a continuación.

\section{Estrategias arquitectónicas}

\subsection{El espacio educador. Bogotá (1998 - 2003):}

Se trata de una pedagogía que enseñaba a los futuros ciudadanos a convivir democráticamente $y$, en este sentido, tenía el reto de lograr tanto el desarrollo de una cultura ciudadana, como una formación académica, propia de todo colegio de educación básica y secundaria. Así se entendió desde la alcaldía de Enrique Peñalosa (1998-2001), donde se planteó una estrategia pedagógica específica para los colegios públicos, que cumpliera estos objetivos por medio de la arquitectura. Esta estrategia quedó plasmada en el libro Construyendo pedagogía. Estándares básicos para construcciones escolares (Rivera Realpe \& Asociados, 2000), un texto que sintetizó el desarrollo de la arquitectura y el urbanismo de los colegios públicos de la ciudad a partir de 1998, por medio de la estrategia del Espacio Educador. Esta apela a la reiteración de actividades cotidianas y recorridos del estudiante en los espacios arquitectónicos del colegio, como estrategia de aprendizaje y comportamiento replicable en los espacios exteriores al colegio, es decir, en la ciudad (Ibídem, 14). Por esta razón, el colegio es pensado y diseñado como una "ciudad a pequeña escala", de manera que "la creación de espacios equivalentes a los reales exteriores, facilite los desarrollos de actividades de intercambio, de relación y transacción entre la comunidad escolar" (Ibídem, 16) (Figura 1).

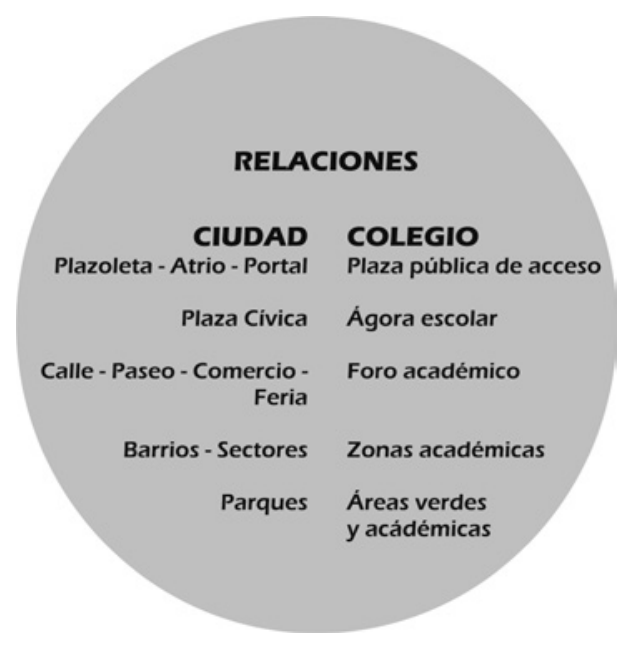

Figura 1 Relaciones ciudad- colegio. Fuente: Rivera Realpe \& Asociados, 2000:12.

Un análisis de las plantas arquitectónicas del Colegio Santiago de las Atalayas (Figura 2), revela cómo la planimetría replica a escala los principales elementos urbano-arquitectónicos de una ciudad: la plazuela, atrio o portal quedan representados en la plaza pública de acceso al colegio, en tanto que la plaza cívica, se convierte en el patio de formación y descanso; asimismo, las calles y paseos urbanos que conectan las viviendas, se transforman en las circulaciones que conectan los salones de clase y áreas académicas, y, finalmente, los parques y áreas verdes propios de toda urbe, son en el colegio, las canchas deportivas y áreas de descanso.

Este enfoque arquitectónico desarrolló en Bogotá los primeros colegios públicos en permitir el uso por parte de la comunidad vecina, abriendo algunas de sus instalaciones en horarios no escolares, aunque paradójicamente se trata de equipamientos que en su implantación tienden a cerrarse a la ciudad, a pesar de querer representarla formalmente al interior. En términos urbanos, se busca retomar el papel del colegio como hito urbano a nivel local y, en este sentido, se propone la recuperación de la imagen institucional del colegio y la generación de espacio público como lugar umbral, de encuentro entre el colegio y la ciudad.

\subsection{Bogotá una gran escuela (2003 - 2008):}

Desde una dimensión urbana, la pedagogía personalista implicó entender una educación a partir de espacios de inclusión, tolerancia y convivencia, por lo que se promovió una visión del colegio como parte integral de la ciudad -y no como un objeto en la ciudad-, que abriera y estrechara lazos con la comunidad, pues para la pedagogía personalista: "La persona es apertura a la comunidad: la persona se funda en una intencionalidad 


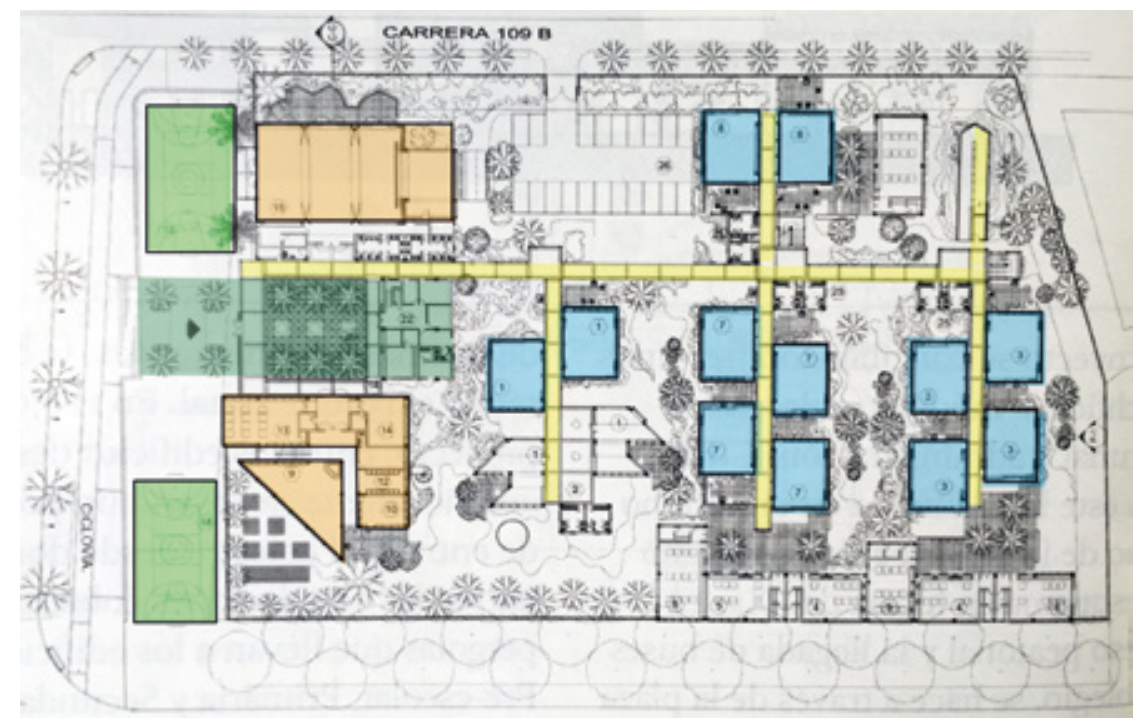

Figura 2 Colegio Santiago de las Atalayas. Bogotá. Arqs. Christian Binkele - Mauricio Pinilla. Fuente: Revista PROA, 2001: 443-445.

que lo hace tender siempre hacia afuera y lo lanza a los otros y al Otro" (Ramírez, 2008: 89).

En el marco de estas ideas, la alcaldía de Bogotá -ahora bajo el mando de Luis Eduardo Garzón (2004-2007)convocó el "Concurso de anteproyectos arquitectónicos para la elaboración del modelo del sistema de diseño para las instituciones educativas distritales". Tal concurso promovía el diseño de una arquitectura escolar que, si bien cumplía con los estándares básicos para construcciones escolares, planteados bajo la anterior alcaldía, tenía dos exigencias particulares: una, requerir a los concursantes el diseño de un sistema arquitectónico adaptable a cualquier lote y, la segunda, generar espacios comunitarios facilitando las relaciones con el entorno (Espinosa, 2009: 54). De esta manera, se buscó que la pedagogía personalista, por medio de la arquitectura, ampliara el rango de acción de la educación por encima de las fronteras de la edificación escolar, haciendo que la presencia física del colegio en la comunidad trascendiera su impacto en la misma. Esta visión de una educación posible más allá de las aulas y del colegio mismo, resulta acorde con los principios básicos de este tipo de pedagogía, pues "para el personalismo, la persona es esencialmente comunitaria y no se puede entender sin lo comunitario. El hombre no alcanza su realización por separado, sino como la expansión armónica de su ser individual y colectivo" (Ramírez, 2008: 90).

Resultado de esta visión, se premiaron 5 propuestas arquitectónicas, todas diferentes $y$, sin embargo, todas comprometidas con la generación de vínculos espaciales con la comunidad, ya fuese por lo permeable de su implantación, por su articulación con el contexto y/o por el diseño de espacios físicos compartibles con los habitantes del sector (Figuras 3 y 4 ).
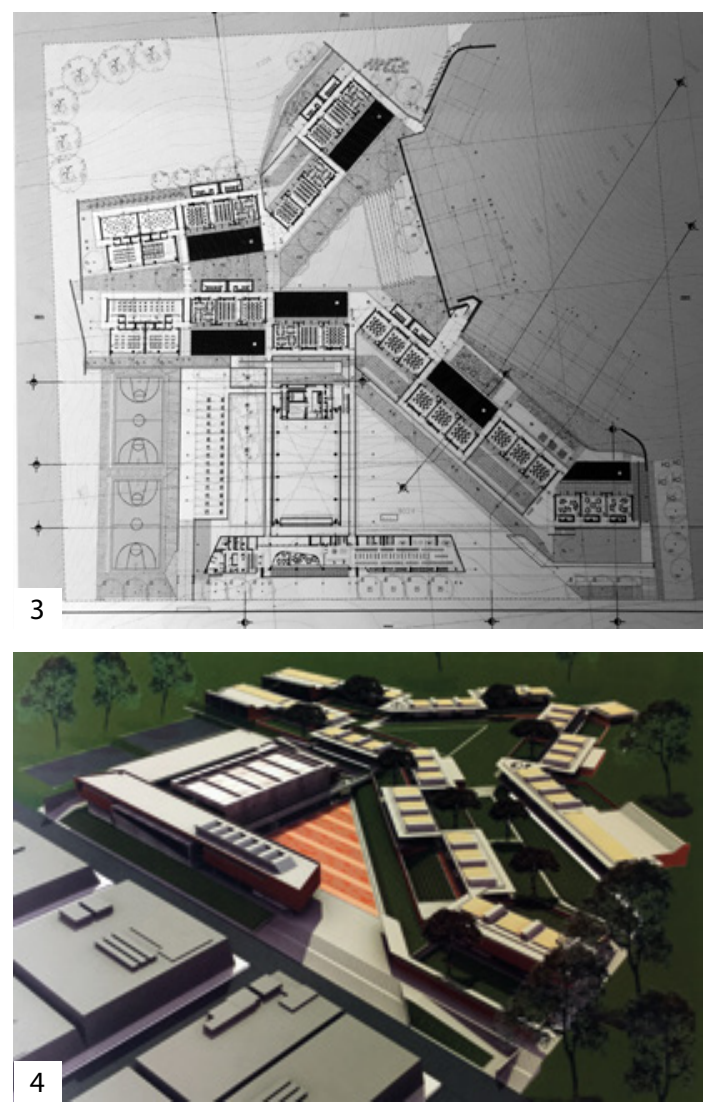

Figura 3 y $42^{\circ}$ Premio Concurso de Anteproyectos I.E.D. Colegio Gabriel García Márquez - Bogotá. Arq. Sergio Trujillo et al. Fuente: Espinosa, 2009: 109-110. 


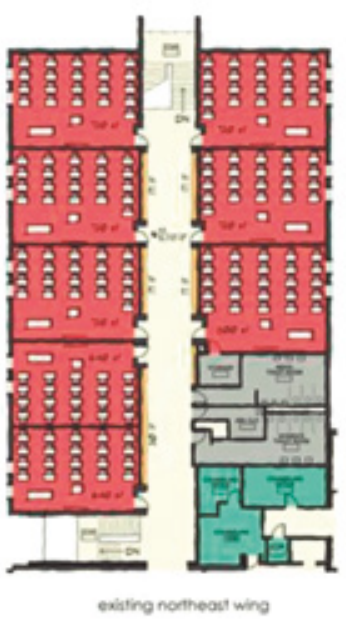

"DE

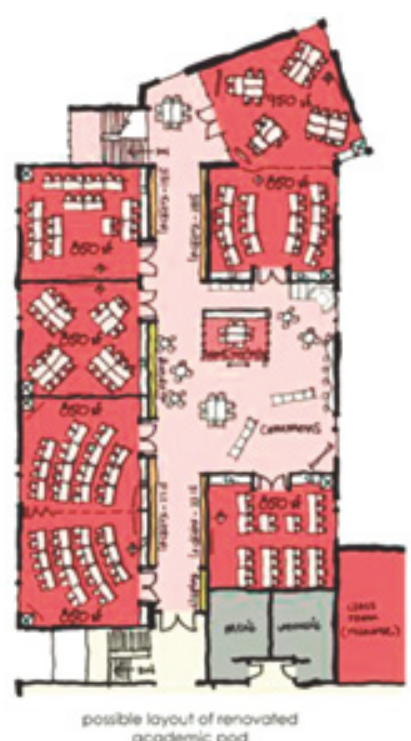

ocodemic pod

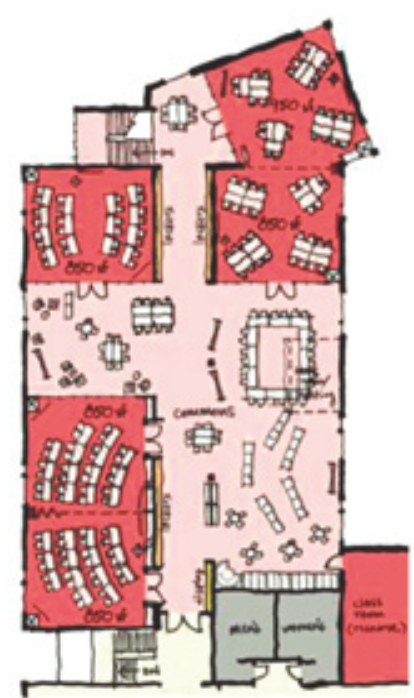

perensol tor mare forbility

Figura 5 "De.......A". Fuente: Ambientes de aprendizaje para el siglo XXI. Concurso público de anteproyectos arquitectónicos para el diseño de colegios y jardines infantiles distritales en Bogotá D.C. Bases del Concurso, 2015

3.3 Ambientes de aprendizaje. Bogotá (2012 - 2015):

Bajo la administración del alcalde Gustavo Petro (2012-2015) se profundiza aún más la política social en la arquitectura educativa, a través del concepto de "ambientes de aprendizaje". Una pedagogía que integra una dimensión de carácter espacial, esto es el "ambiente", "concebido como construcción diaria, reflexión cotidiana, singularidad permanente que asegure la diversidad, y con ella la riqueza de la vida en relación" (Duarte, 2003: 102) y una dimensión pedagógica, de allí que "la expresión ambiente educativo induce a pensar el ambiente como sujeto que actúa con el ser humano y lo transforma" (Ibídem, 102). Aplicado al caso bogotano, se plantea que es el profesor quien "[...] diseña un ambiente de aprendizaje con una intencionalidad pedagógica, orientada a que el estudiante se sirva de todos los recursos que propone el ambiente para adquirir conocimientos, desarrollar capacidades, habilidades y actitudes que le permitan intervenir satisfactoriamente en los contextos propios de su realidad" (Alcaldía Mayor de Bogotá, 2015:15).

A partir de esta nueva conceptualización pedagógica, se abre en 2015 el "Concurso de Arquitectura Ambientes de Aprendizaje para el Siglo XXI Colegios Públicos y Jardines Infantiles". Si en anteriores concursos se cuestionó la pertinencia del colegio cerrado a la comunidad y a la ciudad, en este se llegó aún más lejos en la influencia de las políticas de gobierno en el campo educativo al poner en discusión, por primera vez, la pertinencia tipológica del aula de clase cerrada. En su lugar, propone una arquitectura abierta y flexible que permita la interacción de distintos espacios divididos no por muros, sino por actividades de aprendizaje, donde lo relevante no es el espacio sino los escenarios que allí se propongan para la adquisición del conocimiento, tal como se explicita en las bases del concurso a través de la Figura 5.

En este sentido, se abren los espacios de las aulas tradicionales (Figura 6), permitiendo la integración espacial $-\mathrm{y}$, por ende, pedagógica- y logrando, en teoría, un ambiente relacional de educación, donde se privilegia la educación a partir de la experiencia y práctica con los objetos de estudio, por encima de la educación fundamentada en el estudio teórico.

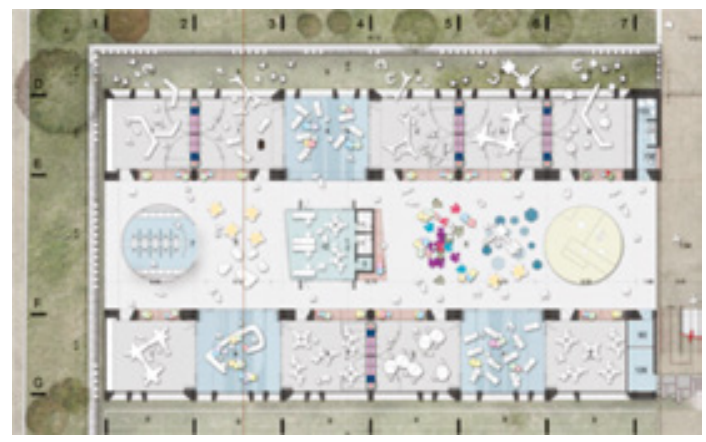

Figura 6 Colegio Pradera El Volcán (detalle). Arqs. Colectivo 720. Fuente: Sociedad Colombiana de Arquitectos - Concurso Ambientes de Aprendizaje 2015 


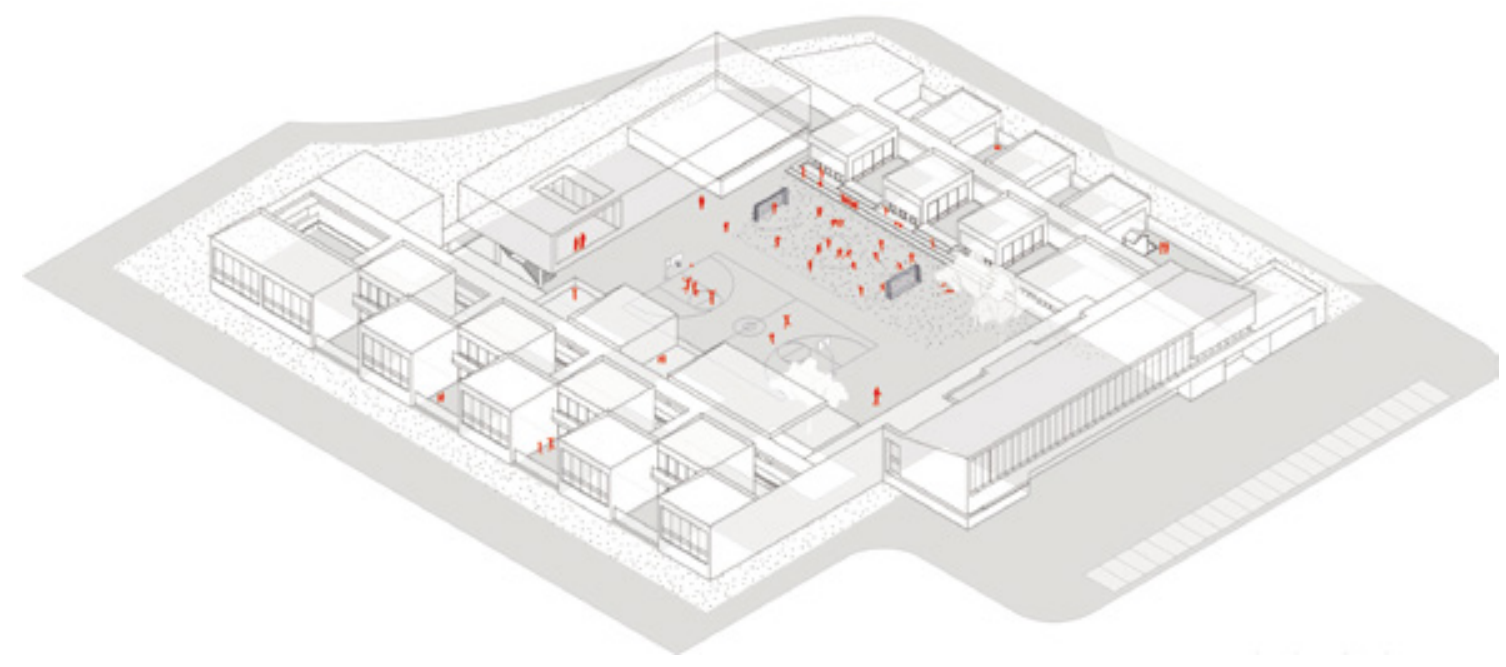

Figura 7 Planta Axonometría - Colegio La Felicidad - Bogotá. FP Arquitectura. Fuente: Sociedad Colombiana de Arquitectos - Concurso Ambientes de Aprendizaje 2015

Los resultados de la aplicación de esta pedagogía aún son inciertos, pues estos colegios se encuentran en proceso de construcción, sin embargo, una imagen del render (Figuras 7 y 8 ) de uno de los proyectos ganadores abre dudas sobre la aplicación efectiva de este modelo autónomo de educación en nuestro medio. Se trata de un modelo en el que el docente aparece como acompañante y orientador de un proceso educativo en el que son los alumnos quienes deciden qué aprender y cómo aprender a partir del ambiente diseñado por el docente para tal fin. Se cuestiona aquí no el modelo pedagógico, sino la pertinencia de este modelo destinado a niños que por su corta edad (6 a 11 años educación primaria y 12 a 16 años, educación secundaria) carecen de la madurez necesaria para decidir autónomamente el desarrollo de un proceso formativo orientado fundamentalmente hacia la actividad y la práctica, y en donde la fundamentación epistemológica de los conocimientos, esto es la teoría, pasaría a un segundo plano.

En todo caso, es importante destacar que no todas las ciudades colombianas han adoptado de la misma manera, ni bajo las mismas estrategias arquitectónicas, los principios pedagógicos personalistas $y$, desde esa perspectiva, vale la pena reconocer la adaptación de estos principios a las necesidades y visiones de las regiones que integran nuestro país, tal como lo propone la Ley General de Educación. Este es el caso de la ciudad de Medellín, donde a partir de los mismos preceptos políticos y pedagógicos, los encargados de la Secretaría de Educación de Medellín y la Empresa de Desarrollo Urbano - E.D.U. han desarrollado unas estrategias alternativas a estos preceptos, en términos de la arquitectura y el urbanismo de los colegios públicos.

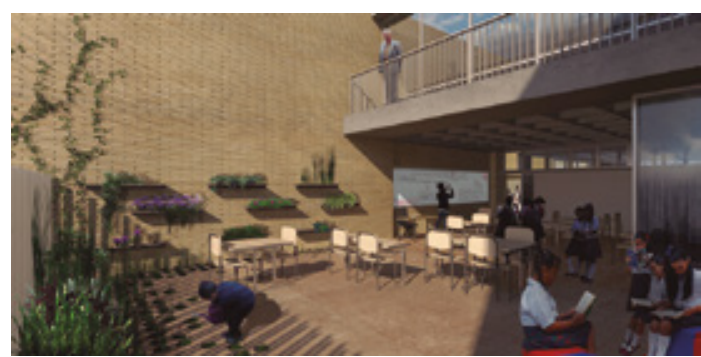

Figura 8 Render interior - Colegio La Felicidad - Bogotá. FP Arquitectura. Fuente: Sociedad Colombiana de Arquitectos. Concurso Ambientes de Aprendizaje 2015

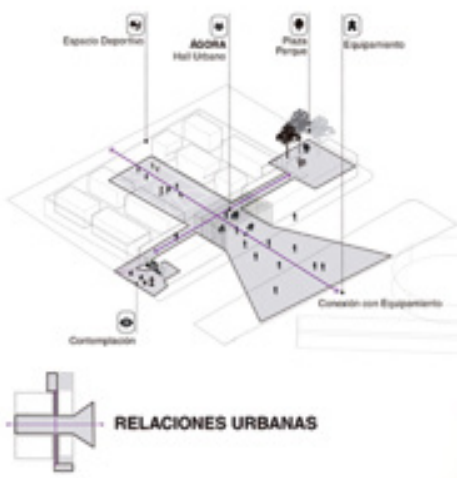

Figura 9 Relaciones urbanas. Colegio Pradera Volcán. Arqs Colectivo 720. Fuente: Sociedad Colombiana de Arquitectos. Concurso Ambientes de Aprendizaje 2015. 


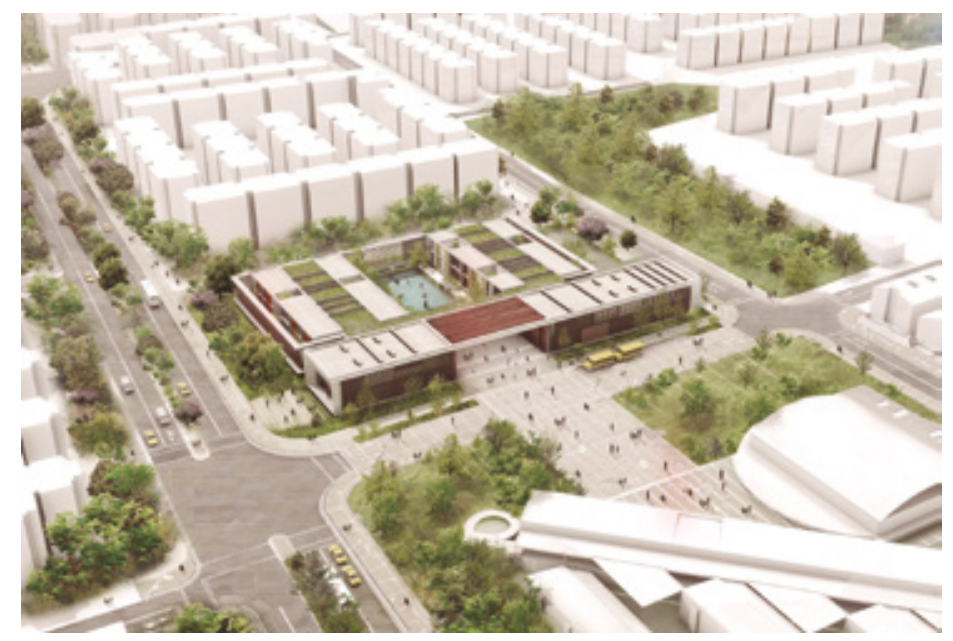

Figura 10 Render. Colegio Pradera Volcán. Args. Colectivo 720. Fuente: Sociedad Colombiana de Arquitectos - Concurso Ambientes de Aprendizaje 2015.
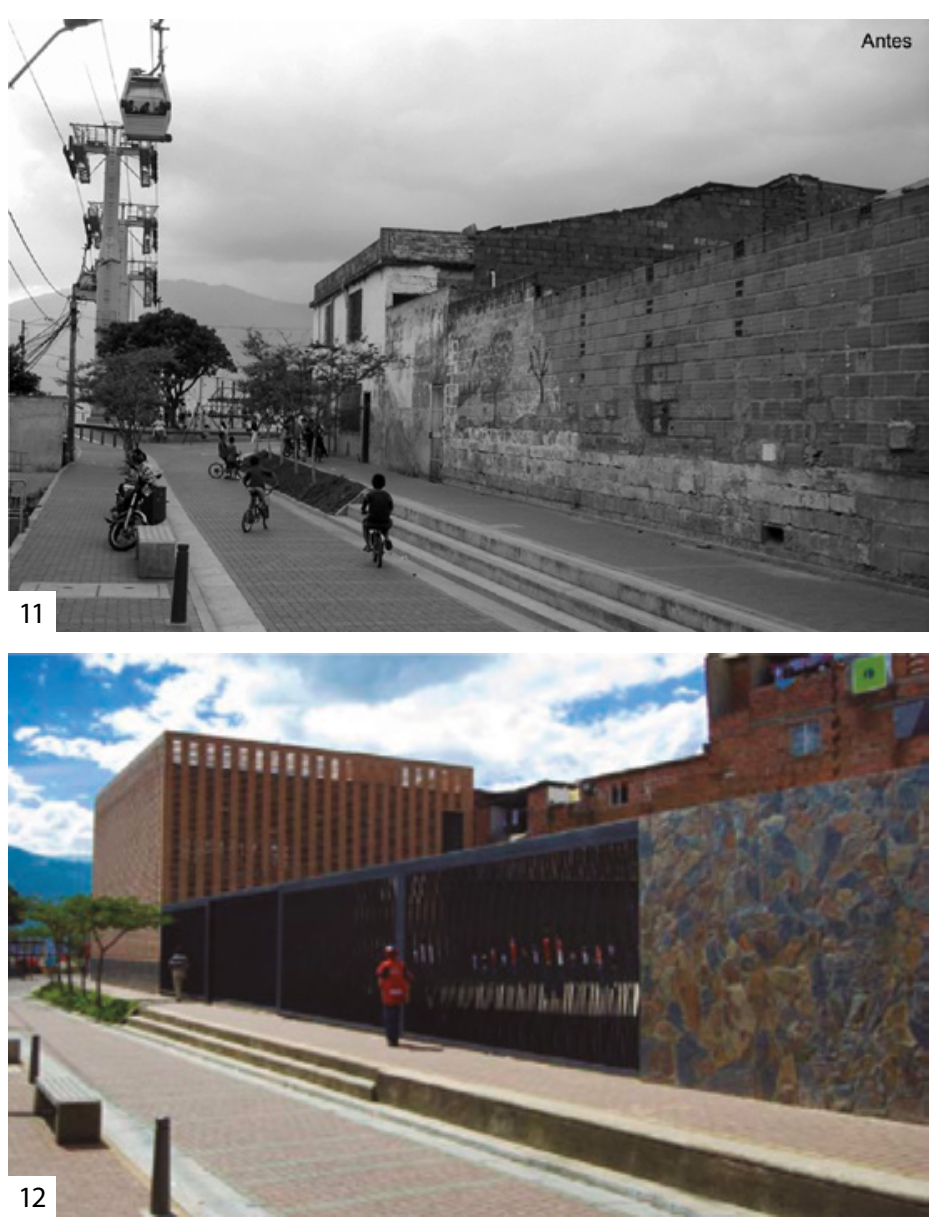

Figura 11 y 12 Institución educativa la CANDELARIA (antes y después) Arqs. Taller de Diseño EDU. Luis Carmona - José Cárdenas. Fuente: Escuela Abierta para la Institución Educativa Integral. Taller de diseño urbano EDU. 


\subsection{Escuela abierta. Medellìn (2004 - 2016):}

Sin duda, es en el departamento de Antioquia donde se ha llevado más lejos el papel del urbanismo en la construcción de una pedagogía educativa en Colombia. La política "Escuela Abierta", propuesta por el alcalde Sergio Fajardo (2004-2007), diluye las fronteras entre colegio y ciudad. De allí el principio fundamental que orienta a la Escuela Abierta:

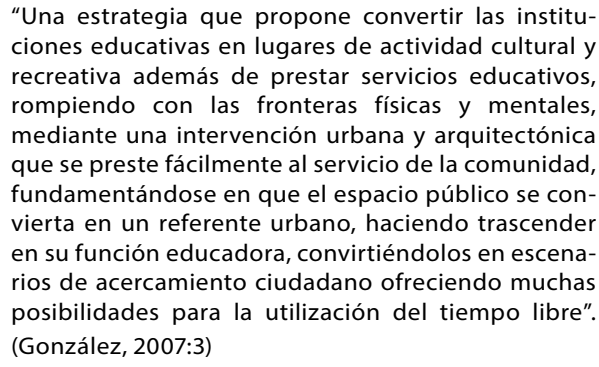
ciones educativas en lugares de actividad cultural y recreativa además de prestar servicios educativos, rompiendo con las fronteras físicas y mentales, mediante una intervención urbana y arquitectónica que se preste fácilmente al servicio de la comunidad, fundamentándose en que el espacio público se convierta en un referente urbano, haciendo trascender en su función educadora, convirtiéndolos en escenarios de acercamiento ciudadano ofreciendo muchas posibilidades para la utilización del tiempo libre". (González, 2007:3)

El desarrollo conceptual de Escuela Abierta y su posterior materialización corrieron a cargo de la Empresa de Desarrollo Urbano E.D.U. (véase Figuras 11 y 12), quienes en una primera fase diseñaron directamente varios de estos colegios. Con posterioridad, las Empresas Públicas de Medellín, a propósito de los 50 años de su fundación, realizaron una importante donación al E.D.U. para la construcción de 10 nuevos colegios, bajo el programa denominado "Colegios de Calidad". Siguiendo el concepto de "Escuela Abierta", estos nuevos establecimientos se destinaban a ser los más representativos de esta visión pedagógica por su diseño y gran inversión económica.

Colegios de calidad (2004 - 2007): En términos generales, los resultados de la política de Medellín aquí expuesta son ambivalentes: si bien es innegable el impacto que en términos de cobertura lograron estos equipamientos educativos en las comunidades donde fueron construidos (véase Figuras 13 y 14), también es cierto que por tratarse de una política experimental que abría indiscriminadamente el colegio a la comunidad, derivó en algunos casos en vandalismo e inseguridad, tal como reconoce Gustavo Restrepo, gerente de proyectos urbanos de la Empresa de Desarrollo Urbano (EDU), cuando afirma: "Estos colegios hacían parte del concepto de aula abierta, ahora sabemos que no es posible y que debemos hacer cerramientos" (El Colombiano, 2008:9). A pesar de esta experiencia, la Gobernación de Antioquia, en manos del otrora alcalde y ahora gobernador Sergio Fajardo (2012-2015), insiste en la validez de los principios de la Escuela Abierta, implementados en esta ocasión desde otro ángulo, y en otros lugares del departamento de Antioquia distintos de su capital, creando nuevos centros educativos en zonas rurales. Este es el caso de los Parques Educativos.

3.5 Parques educativos. Departamento de antioquia (2012 - 2015):

Este modelo pedagógico enfoca sus esfuerzos hacia la construcción de espacios educativos pensados particularmente desde el espacio público, y así lo deja entrever la definición misma de Parque Educativo: "Un Parque Educativo es un espacio público para el encuentro ciudadano en el siglo XXI. Un espacio abierto a toda la comunidad, donde están representados los conceptos fundamentales de Antioquia la más educada" (Gobernación de Antioquia, 2015:191) (Figuras 15 y 16). Desde este enfoque, el rol de la educación adquiere un compromiso mayor, pues sus objetivos se plantean por encima de la formación de competencias académicas, para erigirse en una estrategia de desarrollo económico y social de y para las comunidades, lo que sitúa al programa de Parques Educativos como expresión de una ideología y por ello como "el resultado de una decisión política, de una nueva forma de relacionarse con la población que entiende cada una de sus decisiones como herramienta para avanzar en el desarrollo" (Ibídem, 193).

Lo primero que se advierte al considerar su arquitectura es la ausencia total de barreras para su acceso, salvo las que resguardan las oficinas y salones, por otra parte, destaca la sobriedad y sutileza con la que las arquitecturas de estos equipamientos se insertan en el contexto rural. Se trata de una arquitectura sin formalismos ni alardes estéticos, pero no por ello, carente de calidad, tanto así, que uno de estos proyectos, el Parque educativo Vigía del Fuerte, recibió la máxima distinción en la Bienal Colombiana de Arquitectura (2014).

3.6 Urbanismo pedagógico. Medellín (2012 - 2015):

Esta política del alcalde Aníbal Gaviria (2012-2015) radicaliza el papel del urbanismo en la construcción de una pedagogía educativa en Medellín, por lo que sitúa a la ciudad -y no al colegio- en el centro de este enfoque pedagógico. Sustentado en el principio: "Escuela es todo lo que hay bajo el sol", el Urbanismo Pedagógico conlleva un ambicioso programa destinado a la construcción de proyectos urbano-arquitectónicos realizados en sectores populares con la participación de las comunidades. De esta forma, la metodología de lo participativo ya desarrollada en el caso de los Parques Educativos, se extiende aquí al campo del espacio público, con el fin de generar lugares para el libre encuentro vinculados directamente a equipamientos educativos, y donde se fomente tanto la construcción de lazos sociales como la formación en saberes específicos.

Unidades de vida articulada (U. V. A.): Las U.V.A. son uno de los productos más visibles del urbanismo pedagógico, definidos como infraestructuras que aprovechan la preexistencia de una serie de tanques que dotan de agua potable a los sectores, y alrededor de los cuales se diseña un equipamiento educativo y/o cultural, abierto a toda la comunidad. Se caracterizan por concebir, bajo los principios de la arquitectura participativa y de manera simultánea, el diseño del espacio público como el diseño del equipamiento. Tomar, por ejemplo, "La luz como factor de cohesión" (EPM, 2014), con el fin de consolidar la identidad del equipamiento como hito urbano a nivel local, buscando así su apropiación y cuidado por parte de los ciudadanos (Figuras 17 y 18). 


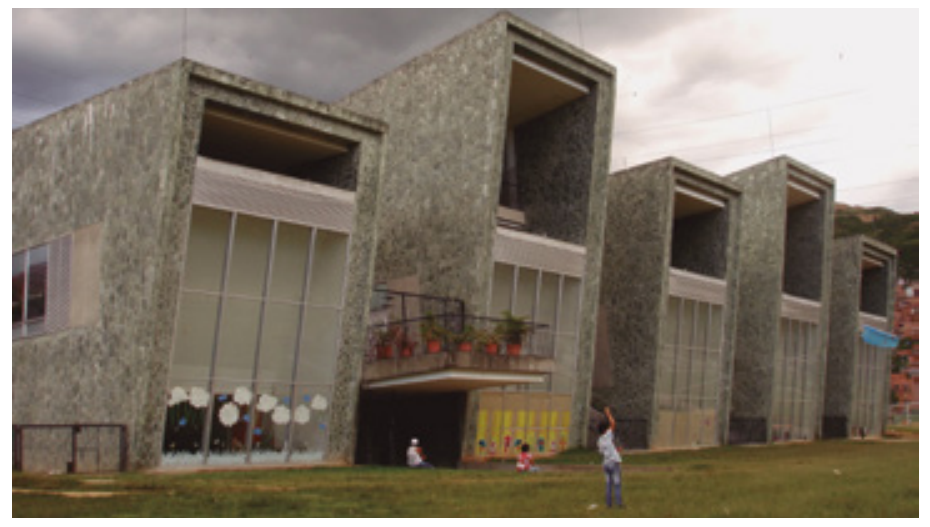

Figura 13 Colegio La Independencia- Medellín Arq. Felipe Uribe.Fuente: Fotografía Arq. William Garcia R.

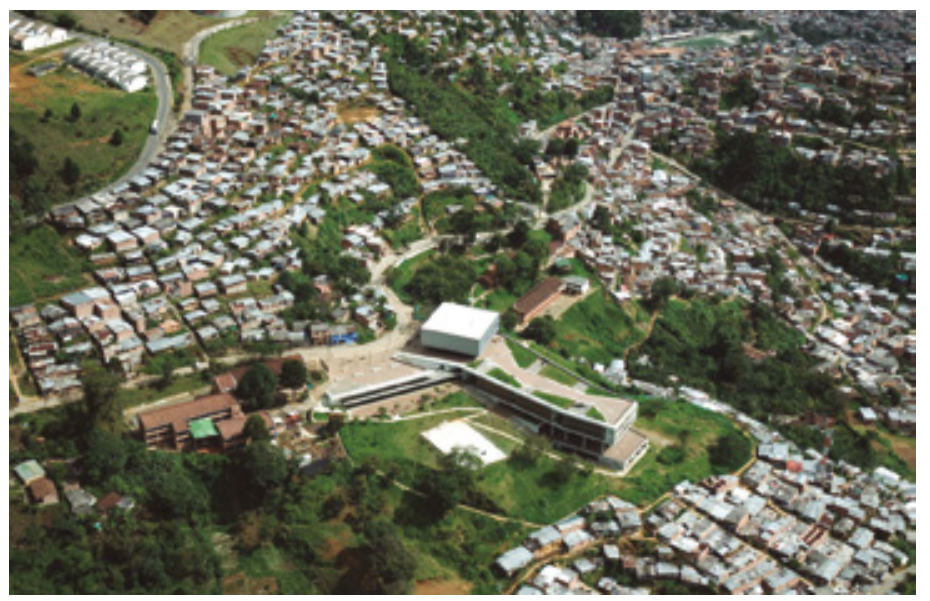

Figura 14 Colegio Santo Domingo - Medellín. Obra Negra Arqs. Fuente: Obra Negra Arqs. Luis Adriano Ramírez.

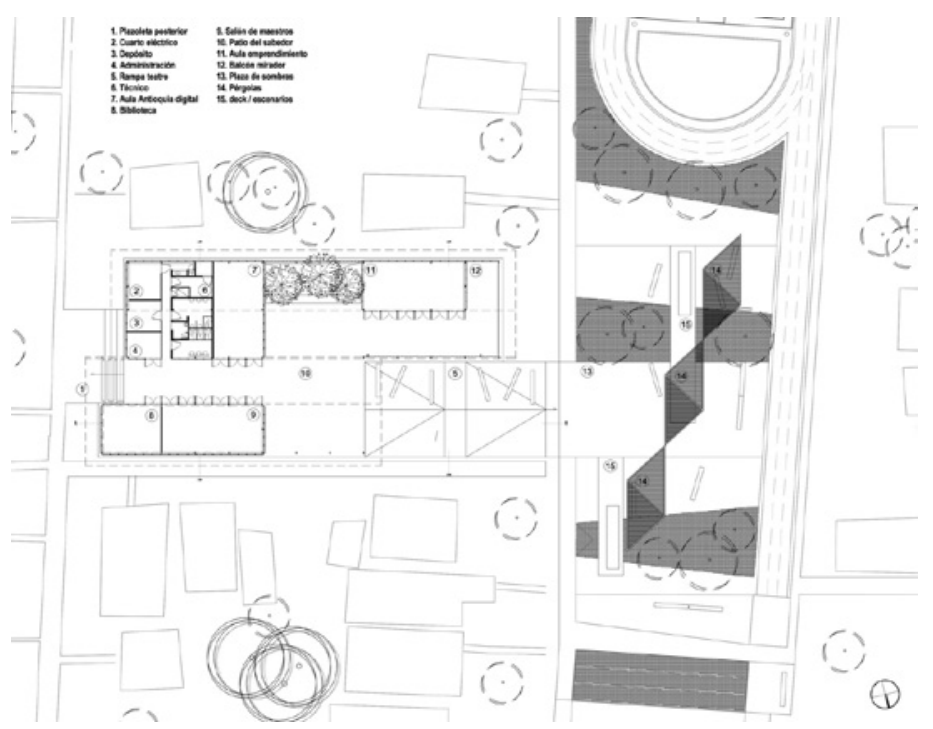

Figura 15 Planta Parque Educativo Vigía del Fuerte - Antioquia. Arqs. M. Valencia D. Herrera, L. Serna, F. Maya. Fuente: Archivo de Farhid Maya. 


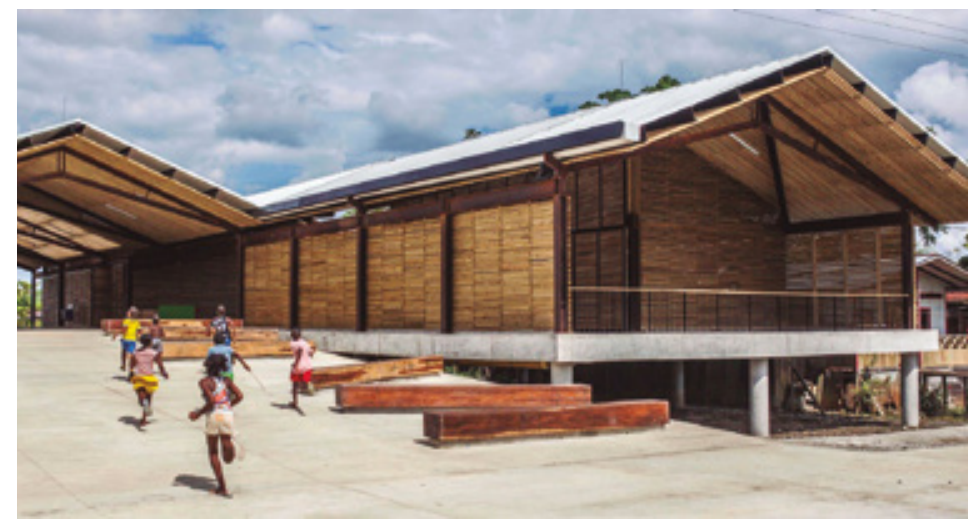

Figura 16 Fotografia Parque Educativo Vigía del Fuerte - Antioquia. Arqs. M. Valencia, D. Herrera, L. Serna, F. Maya. Fuente: Archivo de Farhid Maya.

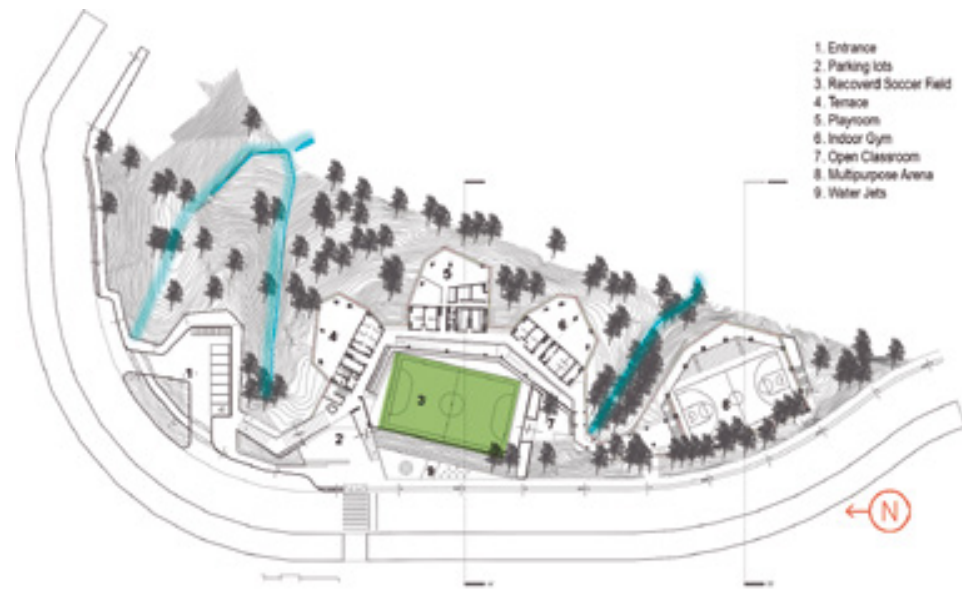

Figura 17 Planta U.V.A. El Paraíso - Medellín. Arq. John Octavio Ortiz et al. Fuente: EDU. Empresa de Desarrollo Urbano.

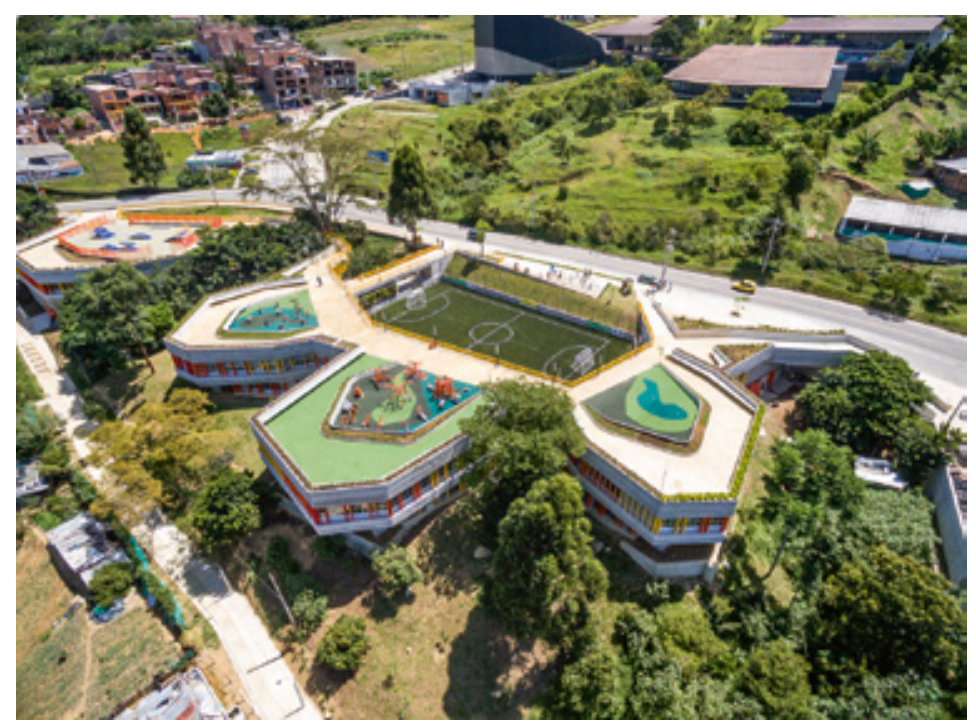

Figura 18 Fotografía U.V.A. El Paraíso - Medellín. Arq. John Octavio Ortiz et al. Fuente: EDU. Empresa de Desarrollo Urbano. 
PEDAGOGÍAS DE UNA IDEOLOGÍA: ARQUITECTURA EDUCATIVA EN COLOMBIA (1994-2016)

ENFOQUE IDEOLÓGICO

IDEOLOGÍA NEOLIBERAL (1994-2016)

(Ley general de Educación. Ley 115 de 1994)

$\begin{array}{lcccc}\text { INSTITUCIÓN } & \text { Alcaldía Mayor de Bogo- } & \text { Alcaldía Mayor de Bogotá } & \text { Acaldía Mayor de Bogotá } & \text { Alcaldía de Medellín / } \\ \text { RESPONSABLE } & \text { tá }(1998-2003) & (2004-2007) & (2012-2015) & (2004-2015)\end{array}$

ENFOQUE PEDAGÓGICO

PEDAGOGÍA PERSONALISTA

ÉNFASIS PEDAGÓGICOS

BASADOS EN

LA PEDAGOGÍA

PERSONALISTA
1. Espacio Educador

2. Bogotá: Una Gran

escuela
3. Ambientes de Aprendizaje Siglo XXI
4. Escuela Abierta

5. Parques Educativos 6. Urbanismo Pedagógico

$\begin{array}{cccc}\text { El Colegio como } & \begin{array}{c}\text { El Colegio como sistema } \\ \text { modular vinculado a la } \\ \text { ciudad }\end{array} & \begin{array}{c}\text { El Colegio como } \\ \text { lugar compartido con la la } \\ \text { ciudad }\end{array} & \begin{array}{c}\text { "Escuela es todo lo que hay } \\ \text { bajo el Sol" }\end{array}\end{array}$

ESTRATEGIAS URBANO ARQUITECTÓNICAS
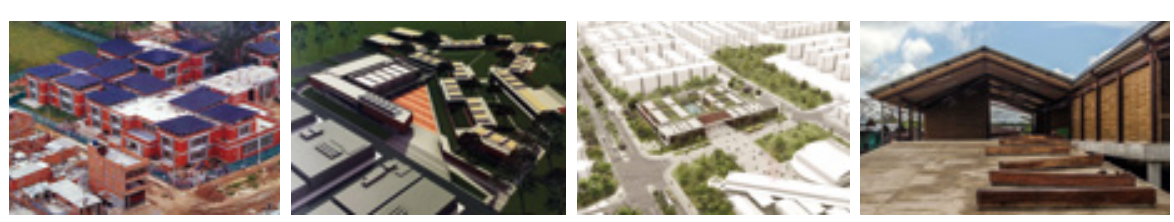

\begin{tabular}{|c|c|c|c|c|}
\hline & $\begin{array}{l}\text { 1. Frente a la } \\
\text { Arquitectura: }\end{array}$ & $\begin{array}{l}\text { 1. Frente a la } \\
\text { Arquitectura: }\end{array}$ & $\begin{array}{l}\text { 1. Frente a la } \\
\text { Arquitectura: }\end{array}$ & $\begin{array}{l}\text { Volumetrías específicas, } \\
\text { vinculadas directamente }\end{array}$ \\
\hline $\begin{array}{l}\text { ESTRATEGIAS } \\
\text { ARQUITECTÓNICAS }\end{array}$ & $\begin{array}{c}\text { Volumetrías específicas } \\
\text { inegradas entre sí, pero } \\
\text { cerradas a la ciudad }\end{array}$ & $\begin{array}{l}\text { Volumetrías prototípicas } \\
\text { integradas entre sí, con } \\
\text { un espacio polivalente } \\
\text { abierto a la ciudad }\end{array}$ & $\begin{array}{c}\text { Volumetrías específicas, } \\
\text { integradas entre sí, con } \\
\text { varios espacios abiertos } \\
\text { y compartidos con la } \\
\text { ciudad }\end{array}$ & $\begin{array}{l}\text { a un espacio público y } \\
\text { compuestas por espacios } \\
\text { flexibles, abiertos e } \\
\text { integrados entre sí, con } \\
\text { todos los espacios abiertos y } \\
\text { compartidos con la ciudad }\end{array}$ \\
\hline
\end{tabular}

ARQUITECTURA QUE
SÍNTESIS CONCEPTUAL
REPRESENTA A LA
CIUDAD


El entramado teórico conceptual que explica la arquitectura educativa pública en Colombia, implicó una lectura de las variables ideológicas, pedagógicas y arquitectónicas que permitiera reconocer la influencia de estas en la arquitectura construida. Esta lectura fue de orden historiográfico y conceptual, lo que permitió identificar cómo no sólo la arquitectura, sino la concepción del ciudadano colombiano del futuro ha ido variando como fruto de esta concepción tripartita, tal como se muestra en la siguiente tabla de resultados (Tabla 1).

\section{CONCLUSIONES}

Los resultados de la investigación permiten constatar cómo la arquitectura educativa pública en Colombia ha pasado lentamente de la introversión arquitectónica, que implicó negar cualquier posible relación del colegio con la ciudad y sus ciudadanos -tal como aconteció la mayor parte del siglo XX-, a la extroversión y apertura espacial que conlleva entender el colegio como un hito urbano cuyas fronteras espaciales y sociales con la ciudad tienden a diluirse cada vez más; una tendencia que en los proyectos más recientes, ha empezado a influenciar uno de los espacios de la arquitectura escolar más tradicionales que había sido inmodificable por muchos años: el aula. La convicción del salón de clases como un espacio confinado de concentración para el aprendizaje de conocimientos, tiende a ser reemplazado por su opuesto: el espacio abierto y polivalente, donde los límites no son muros, sino actividades y donde la silenciosa concentración en los libros se ve reemplazada por el diálogo espontáneo.

En todo caso, estos cambios en la forma y disposición de los espacios educativos, no son solamente la expresión de una ideología y una pedagogía; son, ante todo, la expresión de una concepción política del ciudadano colombiano del siglo XXI, sin duda, una manifestación del proyecto de sociedad y civilización que con la participación de todos los ciudadanos estamos construyendo.
ALCALDÍA MAYOR DE BOGOTÁ. Ambientes de aprendizaje reorganización curricular por ciclos. Secretaría de Educación Dirección de Educación Preescolar y Básica. 2012-2015.

DUARTE, Jakeline. Ambientes de Aprendizaje: Una aproximación conceptual. Estudios Pedagógicos, 2003, n²9, pp. 97-113.

EL COLOMBIANO. Noticias de Medellín, Antioquia y el mundo. Medellín, 2008, p. 9.

EMPRESAS PÚBLICAS DE MEDELLÍN (EPM). Alcaldía de Medellín. Estrategia de Formación Comunidad UVA - Unidades de Vida articulada. Puntos de encuentro para la ciudad. Medellín, 2014.

ESCUELA ABIERTA PARA LA INSTITUCIÓN EDUCATIVA INTEGRAL. Taller de diseño urbano EDU. Documento soporte.

ESPINOSA Parada, Alfonso (ed). Más y mejores colegios para Bogotá proyectos educativos en el marco del convenio de la Secretaría de Educación de Bogotá D.C. y la Universidad Nacional de Colombia. Universidad Nacional de Colombia. Bogotá, 2009.

GOBERNACIÓN DE ANTIOQUIA. Así abrimos la puerta de las oportunidades. Medellín: Imprenta departamental de Antioquia, 2015.

GONZÁLEZ López, José Alonso. Colegios de Calidad propuesta de Escuela Abierta. Boletín externo Empresa de Desarrollo Urbano de Medellín, n 5, 2007.

LEY GENERAL DE EDUCACIÓN. Ley 115 de 1994 República de Colombia. Ministerio de Educación Nacional.

MALDONADO, Rafael. Historia de la arquitectura escolar en Colombia. Bogotá: Universidad Nacional de Colombia, 1999.

PLAN MAESTRO DE EQUIPAMIENTOS EDUCATIVOS. Decreto 449 de 2006.

QUICENO, Humberto. Crónicas históricas de la educación en Colombia. Bogotá: Universidad Pedagógica Nacional, 2003.

RAMÍREZ, Edgar. Historia crítica de la pedagogía en Colombia. Bogotá: Ed. El Búho, 2008.

RAMÍREZ, Francisco. Arquitectura y pedagogía en el desarrollo de la arquitectura moderna. Revista Educación y Pedagogía, Universidad de Antioquia, 2009, $\mathrm{n}^{\circ} 54$, p. 31.

RIVERA REALPE \& ASOCIADOS. Construyendo Pedagogía. Estándares básicos para construcciones escolares. Bogotá: Alcaldía Mayor de Bogotá. Secretaría de Educación, 2000. 